\title{
RNA-binding protein Syncrip regulates Starvation-Induced Hyperactivity in adult Drosophila
}

\author{
Wanhao $\mathrm{Chi}^{1,2^{*}}$, Wei $\mathrm{Liu}^{3}$, Wenqin $\mathrm{Fu}^{2}$, Shengqian Xia ${ }^{4}$, Ellie S. Heckscher ${ }^{1,5,6}$, Xiaoxi \\ Zhuang ${ }^{1,2,6^{*}}$, \\ 1 Committee on Genetics, Genomics, and Systems Biology, University of Chicago, \\ Chicago, Illinois, United States of America \\ 2 Department of Neurobiology, University of Chicago, Chicago, Illinois, United States of \\ America \\ 3 Department of Environmental Health, School of Public Health, China Medical \\ University, Shenyang, China \\ 4 Department of Ecology and Evolution, University of Chicago, Chicago, Illinois, United \\ States of America \\ 5 Department of Molecular Genetics and Cell Biology, University of Chicago, Chicago, \\ Illinois, United States of America \\ 6 Grossman Institute for Neuroscience, Quantitative Biology and Human Behavior, \\ University of Chicago, Chicago, Illinois, United States of America \\ *wchi1@bsd.uchicago.edu; xzhuang@bsd.uchicago.edu
}

\begin{abstract}
How to respond to starvation determines fitness. One prominent behavioral response is increased locomotor activities upon starvation, also known as Starvation-Induced Hyperactivity (SIH). SIH is paradoxical as it promotes food seeking but also increases energy expenditure. Despite its importance in regulating fitness, the genetic contributions to SIH as a behavioral trait remains unexplored. Here, we examined SIH in the Drosophila melanogaster Genetic Reference Panel (DGRP) and performed genome-wide association studies. We identified 23 significant loci, corresponding to 14 genes, significantly associated with SIH in adult Drosophila. Gene enrichment analyses indicated that genes encoding ion channels and mRNA binding proteins (RBPs) were most enriched in SIH. We are especially interested in RBPs because they provide a potential mechanism to quickly change protein expression in response to environmental challenges. Using RNA interference, we validated the role of Syp in regulating SIH. Syp encodes Syncrip, an RBP. While ubiquitous knockdown of Syp led to lethality during development, adult flies with neuron specific Syp knockdown were viable and exhibited decreased SIH. Using the Temporal and Regional Gene Expression Targeting (TARGET) system, we further confirmed the role of Syp in adult neurons in regulating SIH. Lastly, RNA-seq analyses revealed that Syp was alternatively spliced under starvation while its expression level was unchanged. Together, this study not only demonstrates genetic contributions to SIH as an important behavioral trait but also highlights the significance of RBPs and post-transcriptional processes in the brain in regulating behavioral responses to starvation.
\end{abstract}




\section{Author summary}

Animals living in the wild often face periods of starvation. How to physiologically and behaviorally respond to starvation is essential for survival. One behavioral response is Starvation-Induced Hyperactivity (SIH). We used the Drosophila melanogaster Genetic Reference Panel, derived from a wild population, to study the genetic basis of SIH. Our results show that there is a significant genetic contribution to SIH in this natural population, and that RNA binding proteins (RBPs) are especially important. Using RNA interference and the TARGET system, we confirmed the role of an RBP Syp in adult neurons in SIH. Further studies using RNA-seq and Western blotting showed that Syp was alternatively spliced under starvation while its expression level was unchanged, highlighting an essential role of post-transcriptional modification.

\section{Introduction}

Animals living in the natural environment often experience periods of starvation, and they have thus developed different physiological and behavioral strategies to respond to starvation [1,2. One well-documented behavioral response is Starvation-Induced Hyperactivity (SIH), that is, animals will increase their locomotor activity upon starvation [3]. SIH has been observed in both flies [4 9] and mammals 10 12], suggesting that this behavior is evolutionarily conserved. From the viewpoint of energy gain and expenditure, SIH seems paradoxical. On one hand, it facilitates food acquisition and energy intake when food is available [7]; on the other hand, it increases energy expenditure and makes starved animals even more vulnerable when food is not available [4. Therefore, genetic dispositions to either too much or too little SIH would impair fitness depending on the environment. However, the genetic contributions to $\mathrm{SIH}$ as a behavioral trait remains unexplored.

Recent studies suggest that SIH is highly regulated. A number of genes have been shown to regulate SIH in adult Drosophila, including genes encoding energy sensors [5 6], neuropeptides/neuropeptide receptors [4] 8, and neurotransmitters 7]. Recently, $d G 9 a$ was shown to regulate SIH in adult Drosophila 9. Gene $d G 9 a$ encodes a histone methyltransferase, suggesting that SIH is also regulated at the epigenetic level. In addition, post-transcriptional modifications, especially alternative pre-mRNA splicing, has been shown to be effective for cells and animals to quickly respond to starvation as well as other stresses 13 15. However, whether or not alternative pre-mRNA splicing plays a role in SIH has not been reported.

In this study, we first set out to determine whether SIH varied in a population of Drosophila melanogaster with a diverse genetic background. We used the Drosophila melanogaster Genetic Reference Panel (DGRP). The DGRP consists of 205 inbred wild-type strains derived from a single population that was collected from Raleigh, North Carolina, USA [16,17]. It is a recently established community resource and has been used to examine the genetic basis of more than 60 quantitative traits 18 . We quantified SIH in DGRP and confirmed a significant genetic contribution to SIH. We then performed genome-wide association studies and identified 23 significant loci from 14 genes associated with SIH. We found that genes with ion channel activities and mRNA binding activities were especially enriched in SIH. Using RNA interference, we validated the role of a gene encoding an RNA-binding protein Syncrip (Syp) 19] in neurons in regulating SIH. Using the Temporal and Regional Gene expression Targeting (TARGET) system [20], we further confirmed the role of Syp in adult neurons in regulating SIH. Lastly, using RNA-seq, we found that Syp was alternatively spliced under starvation while its expression level remained unchanged. 


\section{Results}

\section{Natural variation in Starvation-Induced Hyperactivity across DGRP}

To study the natural variation in Starvation-Induced Hyperactivity (SIH), we monitored the locomotor activity of 198 strains in the Drosophila melanogaster Genetic Reference Panel (DGRP) 16 17 under either food or starvation conditions in the Drosophila Activity Monitor for three days (S1 Fig, See Methods). DGRP consists of 205 inbred strains. We excluded those that did not breed well.

The locomotor activity of each strain under either food or starvation conditions was plotted as a function of time, sixty hours in total. Representative plots are shown in Fig 1A. We observed that, under the 12-hr:12-hr day-night condition and with the presence of food, all strains showed typical bimodal activity peaks, a day-night rhythm governed by the circadian system [21] (black lines in Fig 1A). Such rhythmic activities were disrupted by food deprivation in most strains. Starved flies became persistently active and the hyperactivity could occur in either day or night (red lines in Fig 11A). There were exceptions as some strains did not show obvious SIH (See a representative strain in Fig $1 \mathrm{~A}$, the bottom panel).

To quantify SIH, we used the total activity during the 12-hr nighttime or daytime, and then calculated the activity difference (Delta Activity, DA) between the starvation condition and the food condition (See Methods for details). Since activities were recorded for a total of sixty hours (3 nights and 2 days), a total of five DA values were obtained accordingly. To compare the DAs among 198 trains in the DGRP, we used the largest DA among five DAs. DA is the absolute activity difference from baseline. We did not use the activity ratio over baseline because we did not see a correlation between DA and baseline activity ( $\rho=0.046, P=0.519$; S1 Table), suggesting that DA was not increased proportionally to the baseline level. A summary plot of the largest DA (DA hereafter) across 198 strains was shown in Fig $1 \mathrm{~B}$. DA ranged from $93.61 \pm 72.22$ to $2101.91 \pm 150.76$ with a broad sense heritability of $H^{2}=0.38$ (S1 Table, S2 Table). Thus, SIH varies among DGRP strains and it has a strong genetic basis.

\section{Starvation-Induced Hyperactivity is negatively correlated with Starvation Resistance}

Previous studies showed that flies with reduced SIH survived longer under starvation 5,6, suggesting that SIH is negatively correlated with Starvation Resistance (SR). To examine whether this relationship could also be observed in DGRP strains which were derived from a natural population, we did a correlation analysis between SIH and SR. We found that SIH was indeed negatively correlated with SR, but the correlation was not particularly strong $(\rho=-0.231, P=1.04 \mathrm{e}-03 ; \mathrm{n}=198)$. Studies have demonstrated that SIH resembles foraging behavior 7]. Previously, we have studied the survival rates of DGRP strains in a foraging environment 22]. Thus, we further examined the correlation relationship between SIH and foraging survival rates of DGRP strains. We did not find any correlation between them $(\rho=-0.073, P=0.309 ; \mathrm{n}$ $=197$ ), which is not too surprising as the beneficial or detrimental effects of SIH may well depend on the specific environmental conditions.

\section{GWA analysis of Starvation-Induced Hyperactivity}

We next performed genome-wide association analysis using the DGRP analysis pipeline (http://dgrp2.gnets.ncsu.edu) 16 17. In total, we identified 23 SNPs/Indels that were associated significantly with SIH $\left(P<1 \times 10^{-5}\right.$, Fig 2 A, Table 1 , S2 Fig, S3 Table. 

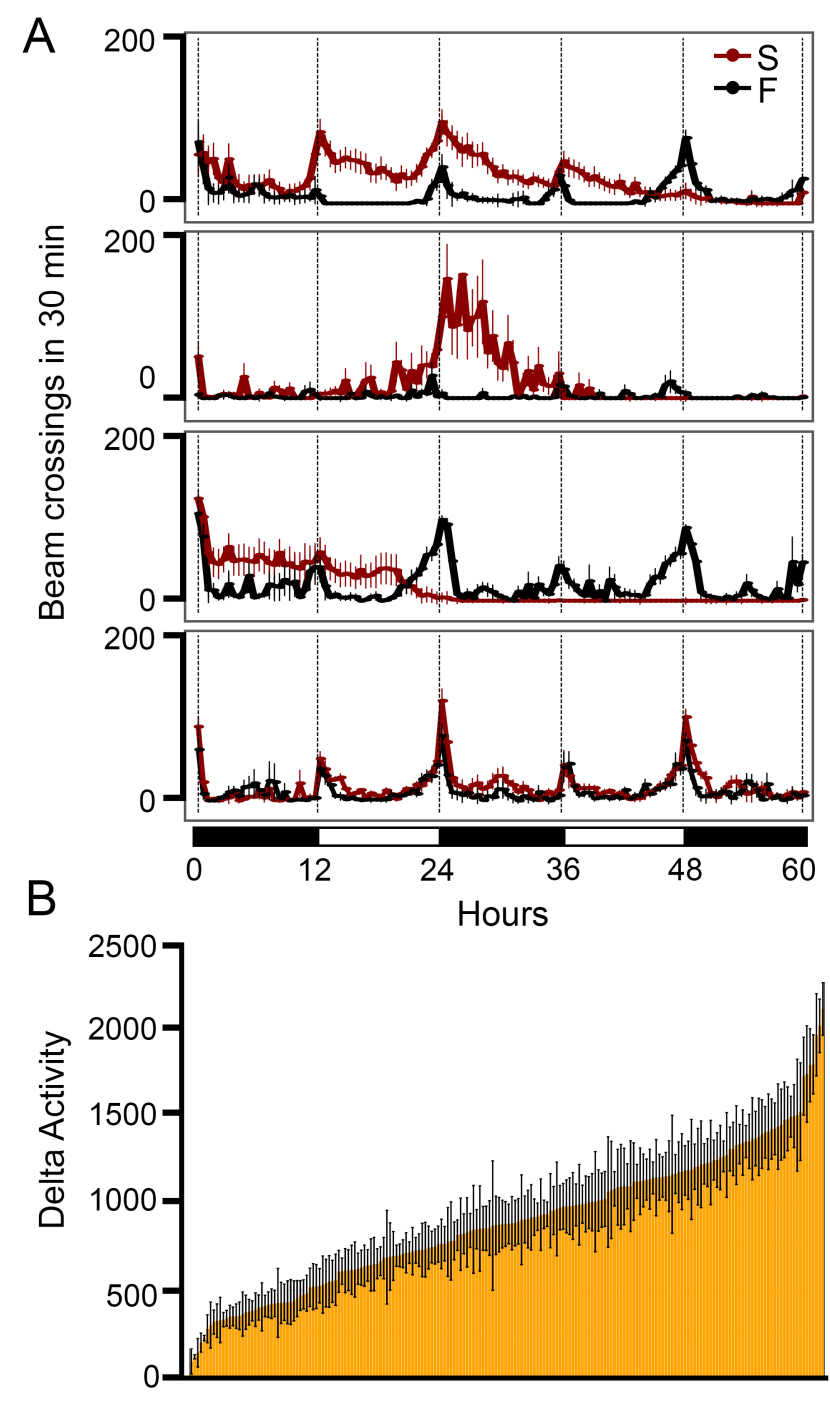

DGRP Strains

Fig 1. Natural variation of Starvation-Induced Hyperactivity in the DGRP. (A) Representative plots of locomotor activity responses to starvation. Beam crosses in 30 min were plotted as a function of time. Black lines represent activities under the food condition (F) and red lines represent activities under the starvation condition (S). Black and white bars at the bottom of panel A represent night and day cycles, respectively. Each plot was from one DGRP strain $(n=8$ per condition). (B) Natural variation occurred in SIH across DGRP. Error bars represent SEM.

Among all these significant loci, $43.5 \%$ of them are located in the intronic region, $43.5 \%$ are in the intergenic region or less than $1 \mathrm{~kb}$ downstream or upstream of an annotated gene and $13.0 \%$ are in the coding region (Fig $2 \mathrm{~B}$ ), which is comparable to the distribution of SNPs/Indels from previous studies in the DGRP $(P=0.4428$, Fisher's exact test) 23. 
bioRxiv preprint doi: https://doi.org/10.1101/2020.01.07.897652; this version posted September 24, 2020. The copyright holder for this preprint

(which was not certified by peer review) is the author/funder, who has granted bioRxiv a license to display the preprint in perpetuity. It is made available under aCC-BY 4.0 International license.

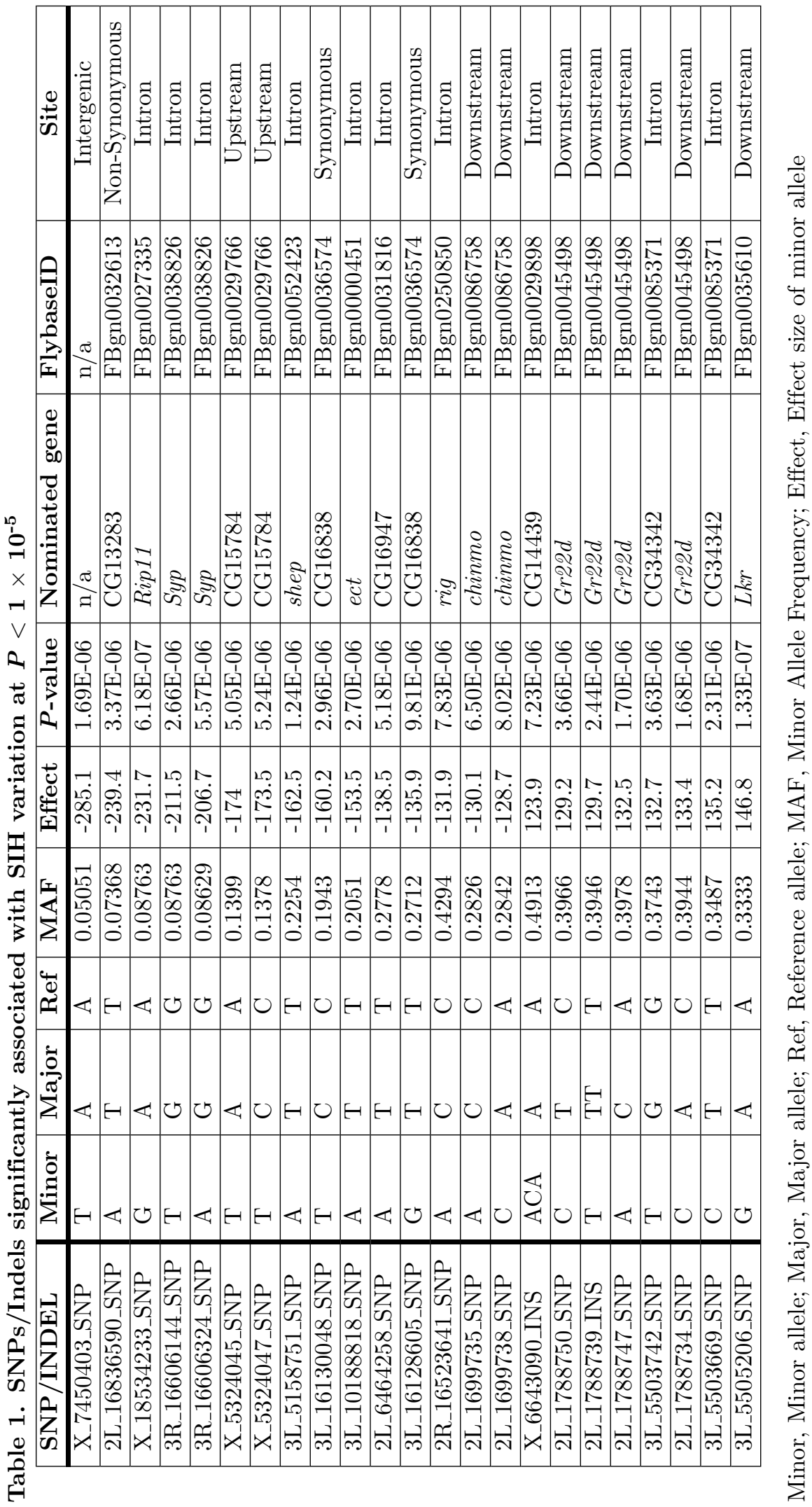




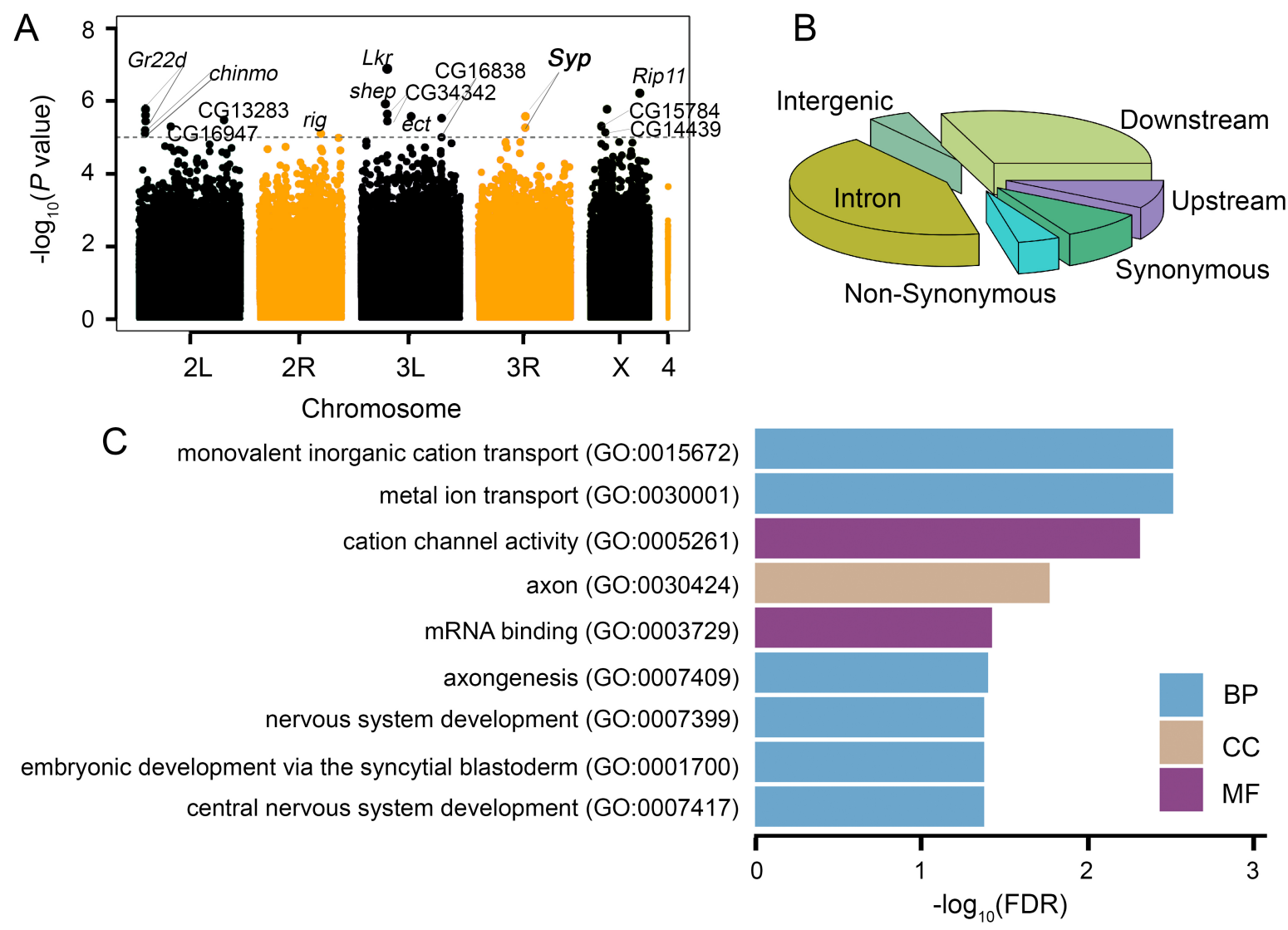

Fig 2. Manhattan plot for GWA, genomic location of significant SNPs, and Gene Ontology (GO) analysis. (A) Manhattan plot of SIH. The gray dotted line is the significant cutoff line used for gene nomination $(P<1$ $\times 10^{-5}$ ). (B) Genomic location of significant SNPs/Indels. (C) GO terms associated with more than 4 genes. BP: Biological Process; CC: Cellular Component; MF: Molecular Function.

\section{Genes associated with SIH are enriched in molecular function GO terms related to ion channels and mRNA binding}

A total of 14 genes were nominated (Table 2). To study if a particular molecular function was enriched in SIH, we performed gene enrichment analysis 24, 25]. Since 14 candidate genes with $P<1 \times 10^{-5}$ in GWA were too few for gene enrichment analyses, we therefore relaxed the cutoff threshold and did a step-wise relaxation using different cutoffs in the range of $P<1 \times 10^{-5}$ and $P<1 \times 10^{-4}$. The number of candidate genes associated with $P<2 \times 10^{-5}, P<4 \times 10^{-5}, P<6 \times 10^{-5}, P<8 \times 10^{-5}$, and $P<1$ $\times 10^{-4}$ is $27,42,63,89$, and 112 , respectively (S4 Table). We performed GO analyses for candidate genes from the cutoffs of $P<1 \times 10^{-4}$ and $P<8 \times 10^{-5}$ since the other cutoffs yielded too few candidates for GO analyses. We found that candidate genes were enriched in 54 and 27 GO terms, respectively (S5 Table). Terms associated with more than four candidate genes with the cutoff of $P<1 \times 10^{-4}$ were shown in Fig $2 \mathrm{C}$. Among them, molecular function GO terms are cation channel activity (GO:0005261) and mRNA binding (GO:0003729). It is worth noting that mRNA binding (GO:0003729) was also associated with candidate genes with the cutoff of $P<8 \times 10^{-5}$ (S5 Table), suggesting that genes with this molecular function are particularly important for SIH.

In addition to GO analysis, we also grouped all significant candidate genes into five categories based on their known or predicted molecular functions from the fly database 
Table 2. Known or predicted molecular functions of nominated genes

\begin{tabular}{|l|l|l|}
\hline Gene & Molecular Function & Category \\
\hline CG34342 & fatty acyl-CoA reductase (alcohol-forming) activity & Metabolic enzyme activity \\
\hline CG13283 & metalloendopeptidase activity & Metabolic enzyme activity \\
\hline CG16838 & DNA binding activity & Nucleic acid binding activity \\
\hline shep & mRNA binding; RNA binding & Nucleic acid binding activity \\
\hline Syp & mRNA binding; RNA binding & Nucleic acid binding activity \\
\hline chinmo & nucleic acid binding & Nucleic acid binding activity \\
\hline rig & protein binding & Protein or small molecule binding activity \\
\hline Rip11 & Rab GTPase binding & Protein or small molecule binding activity \\
\hline CG16947 & ubiquitin-protein transferase activity; Zinc ion binding & Protein or small molecule binding activity \\
\hline Lkr & leucokinin receptor activity; Neuropeptide Y receptor activity & Receptor activity \\
\hline Gr22d & taste receptor activity & Receptor activity \\
\hline CG14439 & n/a & Unknown function \\
\hline CG15784 & n/a & Unknown function \\
\hline ect & n/a & Unknown function \\
\hline
\end{tabular}

(Table 2). Category A includes genes encoding proteins with metabolic enzyme activities (2/14); Category B includes genes encoding proteins with nucleic acid (both DNA and RNA) binding activities (4/14); Category C includes genes encoding proteins with binding activities to proteins or small molecules (3/14); Category D includes genes encoding proteins with receptor activities $(2 / 14)$. Lastly, Category E includes genes with still unknown molecular functions $(3 / 14)$.

We were most intrigued by genes encoding proteins that have mRNA binding activities since many mRNA binding proteins (RBPs) are splicing regulators for alternative pre-mRNA splicing, one of the important cellular responses that are often observed when cells/organisms are under starvation as well as other stresses [13 15]. Two genes that encode RNA binding proteins were shown in the list of nominated genes: Syp (Syncrip) and shep (alan shepard). We chose to validate gene Syp as the loss of shep, even only in neurons, can lead to abnormal locomotor activities in adult flies [26, which could complicate the final data interpretation.

\section{Knockdown of Syp in neurons significantly affects SIH}

To test whether gene Syp plays a role in SIH, we used the binary UAS/GAL4 system [27] to knock down its expression. We first crossed the gene-specific UAS-RNAi line with an actin-Gal4 driver to generate ubiquitous Syp knockdown flies. We observed that ubiquitous Syp knockdown led to lethality before the adult stage. This observation is consistent with previous studies showing that Syp mutant flies have highly decreased adult viability $[28$. Given that Syp is highly expressed in adult heads

(modENCODE.org), we thus generated neuron-specific Syp knockdown flies by crossing UAS- Syp RNAi flies with a pan-neuronal driver $n S y b$-Gal4. Neuron-specific Syp knockdown flies survived well, suggesting that the fatality of ubiquitous Syp knockdown flies is likely due to the lack of expression of Syp in non-neuronal cells.

We then tested neuron-specific Syp knockdown and control flies in the Drosophila Activity Monitor under either food or starvation conditions. As shown in Fig 3A, neuron-specific Syp knockdown did not affect the locomotor activities in the food condition, suggesting that Syp in neurons is not required for the general locomotor activity. However, Delta Activity from them was significantly lower than that from control flies (Fig $3 \mathrm{~B}$ ), demonstrating a specific role of Syp in SIH. The reduced Delta Activity was also observed in neuron-specific Syp knockdown flies generated from 
A

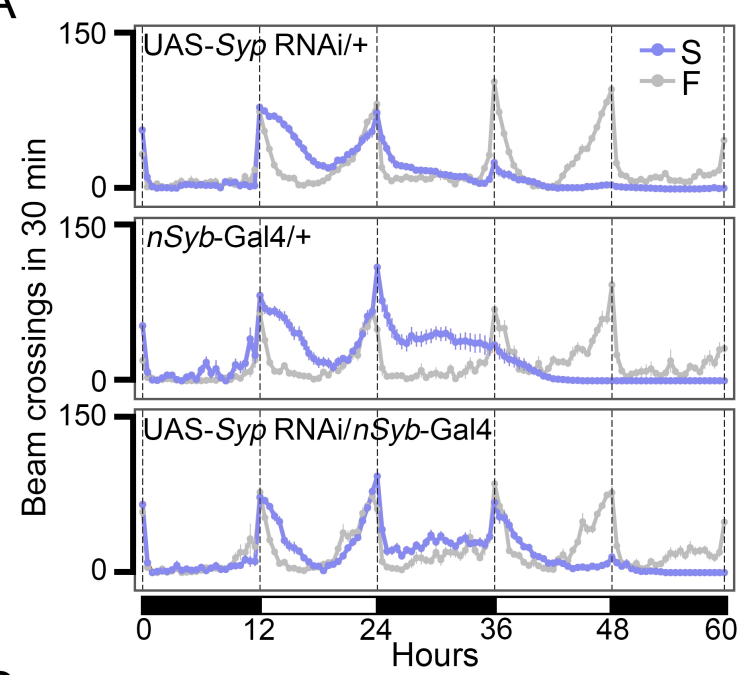

C

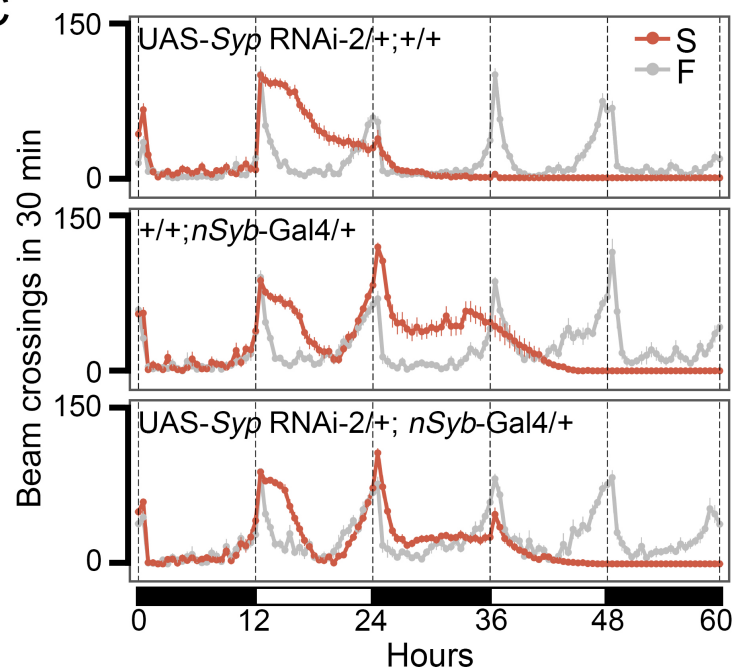

another independent UAS- Syp RNAi line (Fig 3C and 3D). We therefore conclude that neuronal specific knock down of Syp impairs SIH.
141

$B$
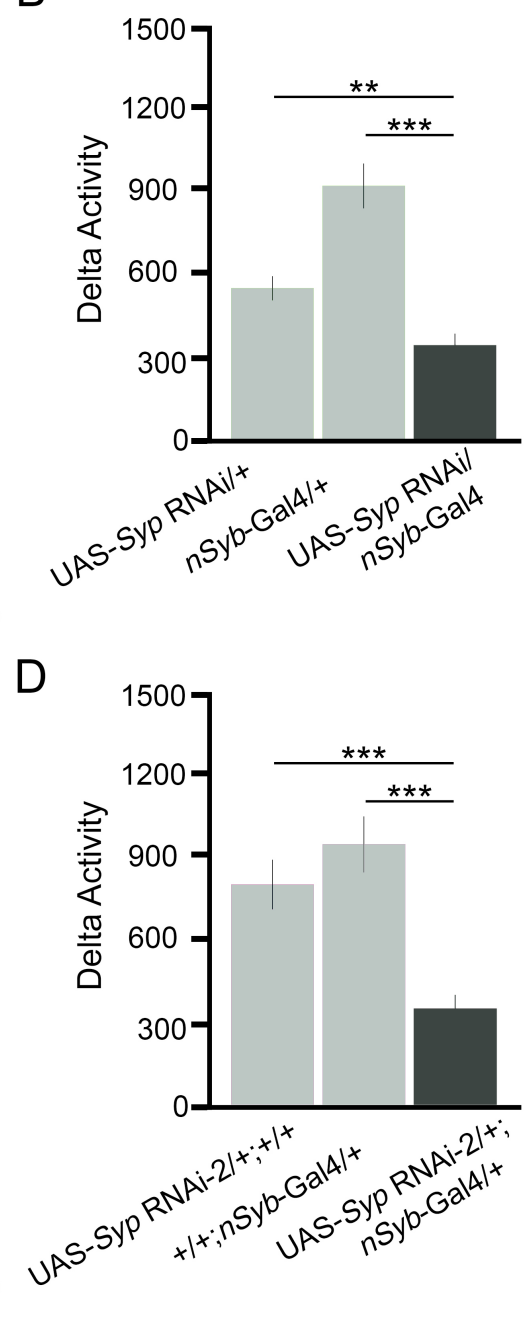

Fig 3. Knocking down of Syp in neurons reduces SIH. (A) Locomotor activity responses to starvation from neuron-specific Syp knockdown flies (genotype: UAS-Syp RNAi/nSyb-Gal4) and two parental control lines (genotypes: UAS-Syp RNAi/+ and nSyb-Gal4/+, respectively). Gray lines represent activities under the food condition (F) and purple lines represent activities under the starvation condition (S). Black and white bars at the bottom of the panel represent night and day cycles, respectively. $\mathrm{n}=16-20$ per genotype per condition. (B) Summary plot of SIH in panel A. (C) Locomotor activity responses to starvation from neuron-specific Syp knockdown flies generated from a second RNAi line (genotype: UAS-Syp RNAi-2/+; $n S y b-G a l 4 /+$ ) and two parental control lines (genotypes: UAS-Syp RNAi-2/+; +/+ and $+/+; n S y b$-Gal4/+, respectively). $\mathrm{n}=20-24$ per genotype per condition. (D) Summary plot of SIH in panel C. ${ }^{* *} P$ $<0.01,{ }^{* * *} P<0.001$, unpaired $t$-test with Bonferroni Correction. Error bars represent SEM.

\section{Syp in adult neurons regulates Starvation-Induced Hyperactivity}

Syp has been shown to affect synaptic morphology and vesicle release at the neuromuscular junction in Drosophila larvae [29,30. Moreover, it has also been 
implicated in neuron/glia cell fate determination during development 31,32. The role of Syp in regulating SIH, therefore, could be due to its developmental effects. To rule out the possibility that Syp regulates SIH due to its developmental effects, we combined UAS-Syp RNAi with the Temporal and Regional Gene Expression Targeting (TARGET) system 20] to control the expression of RNAi temporally. The TARGET system consists of three elements: Gal4, UAS, and temperature sensitive Gal80 mutant $\left(\right.$ Gal $\left.80^{\text {ts }}\right)$. The Gal80 $0^{\text {ts }}$ blocks Gal4-induced RNAi expression when tissue is exposed to the Gal $80^{\text {ts }}$ permissive temperature $\left(18^{\circ} \mathrm{C}\right)$. In contrast, at a Gal $80^{\text {ts }}$ restrictive temperature $\left(31^{\circ} \mathrm{C}\right)$, Gal $80^{\text {ts }}$ loses its binding to Gal4, which allows Gal4-dependent RNAi expression. Therefore, breeding flies at $18^{\circ} \mathrm{C}$ and testing them at $31^{\circ} \mathrm{C}$ allowed us to bypass the developmental effects of Syp KD.

We first bred flies at $18^{\circ} \mathrm{C}$ until adult flies emerged. We then shifted the temperature of the incubator to $31^{\circ} \mathrm{C}$ and incubated adult flies in the new food vials for one more day, which was followed by behavioral testing at $31^{\circ} \mathrm{C}$ under both starvation and food conditions for three days (Fig 4A A). Results showed that Tub-Gal $80^{\text {ts }} /+$; $n S y b$-Gal4/UAS-Syp RNAi (ts-Syp-KD) flies exhibited significantly attenuated SIH in comparison to ocntrol flies (genotypes: $+/+; n S y b$-Gal4/+, Tub-Gal80 $0^{\text {ts }} /+$; UAS-Syp RNAi/ + , and Tub-Gal $80^{\text {ts }} /+;+/+$. Fig $4 \mathrm{~B}$ and $4 \mathrm{C}$ ), and that the Delta Activity level from ts-Syp-KD flies was comparable to that from $+/+$; $n$ Syb-Gal4/UAS-Syp RNAi (Syp-KD) flies $(P=0.6863)$, demonstrating that knocking down of Syp in the adult stage is sufficient for suppression of SIH.

To verify that Gal $80^{\text {ts }}$ did inhibit Gal4 and prevent Syp $\mathrm{KD}$ at $18^{\circ} \mathrm{C}$, we also bred and tested adult flies all at $18^{\circ} \mathrm{C}(\mathrm{Fig} 4 \mathrm{D})$. Under this condition, as shown in Fig $4 \mathrm{E}$ and $4 \mathrm{~F}$, ts-Syp-KD exhibited a similar level of SIH to control flies and a significant higher level of SIH than Syp-KD flies. To further confirm that Syp was indeed knocked down in adult flies at $31^{\circ} \mathrm{C}$ but not at $18^{\circ} \mathrm{C}$, we compared the Syp protein levels in adult fly heads from ts-Syp-KD and Syp-KD flies that were maintained at either $31^{\circ} \mathrm{C}$ or $18^{\circ} \mathrm{C}$ for two days. As shown in Fig $4 \mathrm{G}$ and $4 \mathrm{H}$, at $31^{\circ} \mathrm{C}$, the Syp level was comparable between two genotypes $(P=0.4476)$. In comparison, at $18^{\circ} \mathrm{C}$, the Syp level in ts-Syp-KD flies was about $20 \%$ higher than that in Syp-KD flies $(P=0.0496)$. The relatively moderate difference between them is most likely due to non-neurons mixed in the quantification of proteins in the fly head. Taken together, we conclude that it is Syp in adult neurons that regulates $\mathrm{SIH}$.

\section{Syp is alternatively spliced upon starvation}

Gene Syp spans a region of $54 \mathrm{~kb}$ in the genome, and it has 20 well documented splice variants (www.flybase.org, Fig 5). To study how Syp was regulated under starvation, we performed RNA sequencing (RNA-seq) on adult heads from control flies $\left(w^{1118}\right)$ reared under either food or starvation conditions S3 Fig, S4 Fig, S5 Fig, S6 Fig, see Methods for details). We first examined whether the Syp mRNA level was altered after starvation. Results from differential expression analyses showed that a total of 574 genes were down-regulated, and a total of 84 genes were up-regulated under starvation (Criteria applied: false-discovery rate $(F D R)<0.05$ and fold change $\geq 2$ or fold change $\leq-2$; S6 Table. Gene Syp was not on the list, suggesting that its expression was not dramatically regulated by starvation. This conclusion was further corroborated by similar protein levels of Syp under two conditions (S7 Fig). To examine the functional implications of down- versus up-regulated genes, we performed gene enrichment analyses for each list of genes. We found that down-regulated genes participate in a variety of metabolic pathways, whereas up-regulated genes are mainly enriched in pathways related to DNA repair, nutrients recycling, and spliceosome (S6 Fig). The fact that genes involved in the spliceosome are upregulated upon starvation suggests that alternative splicing may underlie responses to starvation. 
A

B

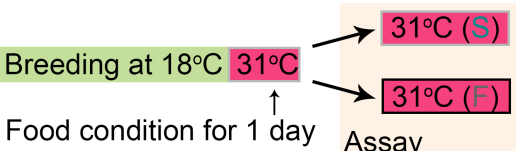

1507 Tub-Gal80ts/+;nSyb-Gal4/UAS-Syp RNAi
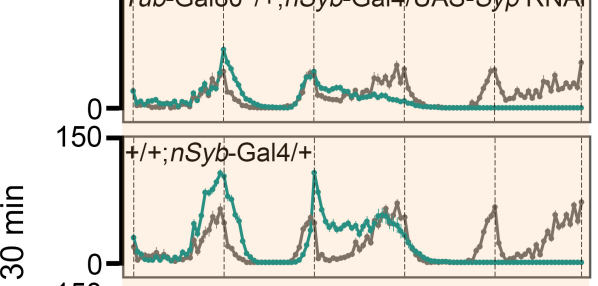

$\subseteq 1507$ Tub-Gal80ts/+;UAS-Syp RNAi/+

क

U
ह
$\searrow$
$\varnothing$

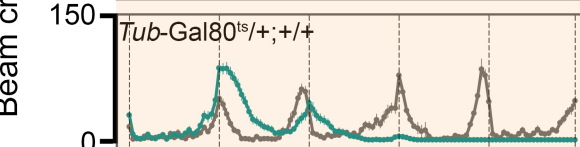

1507 +/+;nSyb-Gal4/UAS-Syp RNAi $-a$ S:

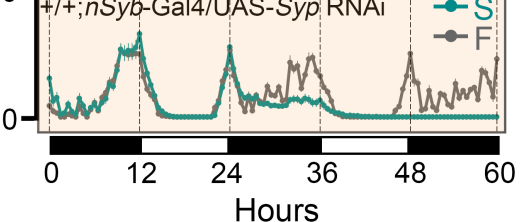

C

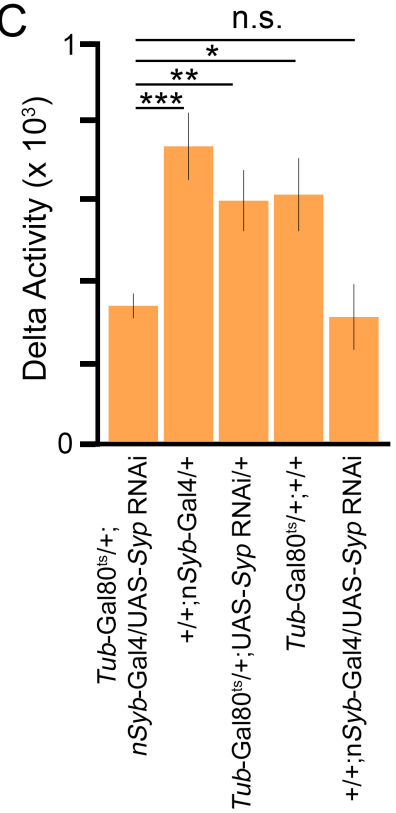

G Tub-Gal80ts/+;

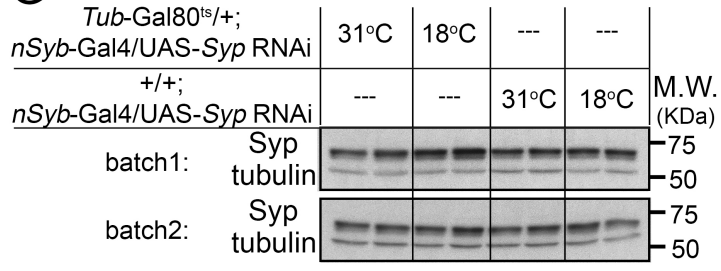

$\mathrm{H}$

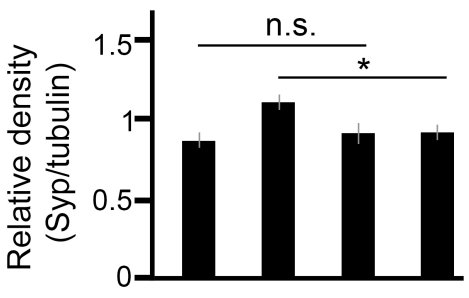

E
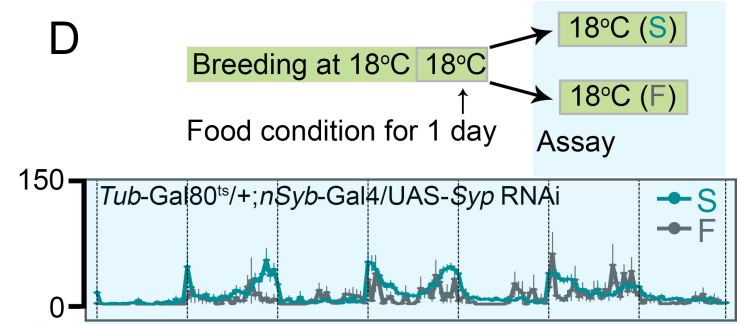

$1507+/+; n S y, b-G a l 4 /+$
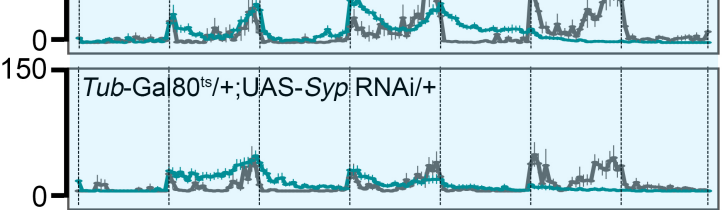

1507 Tub-Gal $80^{\mathrm{ts}} /+;+$ +

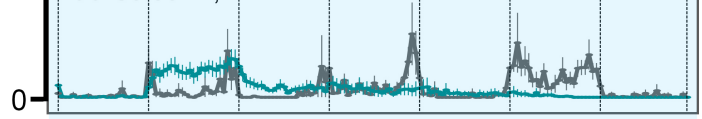

150 +/+;nSyb-Gal4/UAS-Syp RNAi

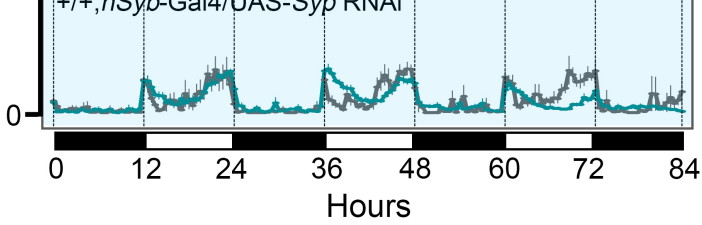

Fig 4. Syp in adult neurons regulates SIH. (A) Breeding and testing scheme. S: starvation condition; F: food condition. (B) Locomotor activity responses to starvation from each genotype under the condition shown in panel A. Gray lines represent activities under the food condition $(\mathrm{F})$ and green lines represent activities under the starvation condition (S). $\mathrm{n}=24-30$ per genotype per condition. (C) Summary plot of SIH from each genotype in panel B. (D) Breeding and testing scheme. S: starvation condition; F: food condition. (E) Locomotor activity responses to starvation from each genotype under the condition shown in panel D. $\mathrm{n}=8-28$ per genotype per condition. (F) Summary plot of SIH from each genotype in panel E. (G) Western blot of adult fly head homogenate from Tub-Gal80 ${ }^{\text {ts }} /+; n$ Syb-Gal4/UAS-Syp RNAi (ts-Syp-KD) and +/+;nSyb-Gal4/UAS-Syp RNAi (Syp-KD) flies. Four biological replicates per genotype per condition. Tubulin is the loading control. (H) Quantification of Syp from four biological replicates. ${ }^{*} P<0.05$, ${ }^{* *} P<$ $0.01,{ }^{* * *} P<0.001$, n.s: $P>0.05$, unpaired $t$-test with Bonferroni Correction. Error bars represent SEM. 


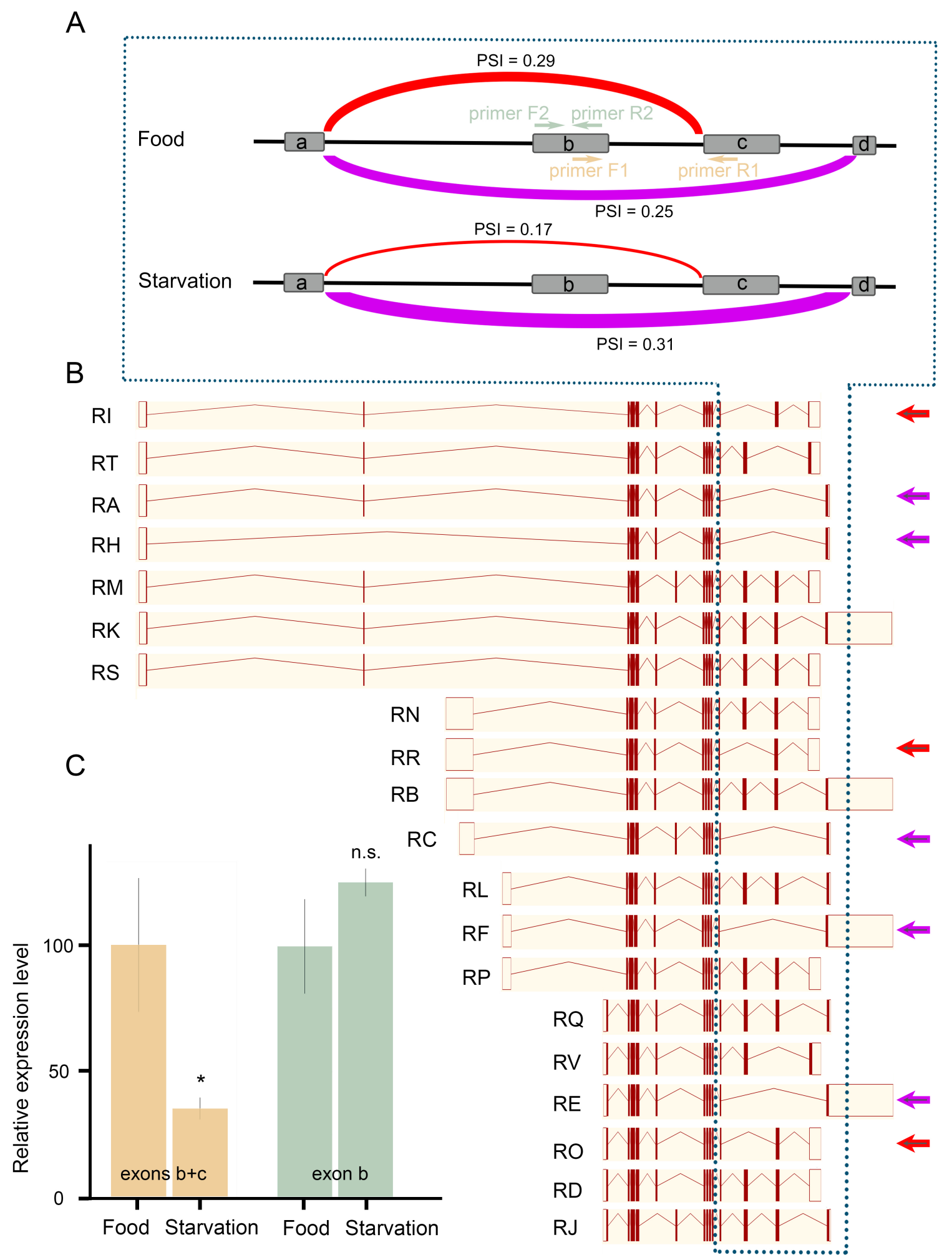

Fig 5. Syp is alternatively spliced under starvation. (A) Differential usage of exons in Syp under food and starvation conditions. Four exons involved in the alternative splicing were shown and referred to as exon a, exon b, exon c, and exon d. PSI: Percentage Sliced In. (B) Various splice variants of Syp (www.flybase.org). Arrows indicate splice variants that were either downregulated (red color) or upregulated (purple) under starvation. (C) Relative expression of targeted exons measured by qPCR. The targeted locations of each pair of primers were indicated in panel A. ${ }^{*} P<0.05$, n.s: $P>0.05$, unpaired $t$-test, $\mathrm{n}=4$ per condition. Error bars represent SEM. 
To identify genes that underwent alternative splicing under starvation, we analyzed RNA-seq data using LeafCutter, a software that can identify and quantify both novel and known alternative splicing events 33 . LeafCutter focuses on intron excisions and groups RNA-seq reads into different clusters based on their mapped location to the genome. Therefore, the final data presentation is in the form of clusters that include various splicing forms. A cluster was considered significant when FDR $<0.05$. A total of 1927 clusters from 359 genes were alternatively spliced under starvation (S7 Table). Gene enrichment analyses showed that these genes were implicated in a variety of biological processes, including regulation of mRNA splicing itself (S8 Table).

Remarkably, the most significant molecular function GO term is mRNA binding (S8 Fig S8 Table, suggesting that upon starvation, genes encode RNA-binding proteins tend to be alternatively spliced.

Among these 1927 significant clusters, 359 of them, from 180 genes, have a $\Delta$ PSI larger than $10 \%$. PSI, Percentage Sliced In, is the fraction of a gene's mRNAs that contain the exon; therefore, $\triangle \mathrm{PSI}$ is the difference in PSI between the starvation condition and the food condition. Among these 359 clusters, $80.8 \%$ of them have small splicing changes $(10 \%<\Delta$ PSI $<25 \%), 18.7 \%$ have intermediate splicing changes $(25 \%$ $\leq \Delta \mathrm{PSI} \leq 50 \%)$, and $0.5 \%$ have large splicing changes $(\Delta \mathrm{PSI}>50 \%)$. Gene Syp is alternatively spliced under starvation (S7 Table). A total of 10 splicing events were found in this significant cluster. Among them, the top two $\Delta$ PSIs were -0.12 and 0.06 (Fig 5A). The splice variants that correspond to the changes are Syp-RI (Transcript ID: FBtr0334711), Syp-RR (FBtr0334720), Syp-RO (FBtr0334717), Syp-RA (FBtr0083958), Syp-RH (FBtr0113256), Syp-RC (FBtr0083960), Syp-RF (FBtr0083961), and Syp-RE (FBtr0083963) (Fig 5B).

To confirm that these changes were not from sequencing noises, we designed PCR primers to target the middle exons within this region (exons b and c, Fig 5A A) and performed RT-qPCR. Results showed that the expression of exons b and c in starved flies was reduced to $38 \%$ of that in flies maintained under the food condition (Fig 5 C), which confirmed the increased splicing from exon a to exon d. We further designed primers to target exon $\mathrm{b}$ only and found that the expression of exon $\mathrm{b}$ was unchanged after starvation (Fig $5 \mathrm{C}$ ), which was not surprising since this exon was involved in two splicing events that were regulated in opposite directions by starvation. Therefore, Syp was alternatively spliced upon starvation. It is thus specific Syp splice variants that play the role in regulating SIH.

In order to examine the significance of alternative splicing involving exons b and c, we generated exons b and c deletion flies using CRISPR/Cas9 34,35. We designed sgRNAs to target both exons (S9 Fig). Complete deletion of exons b and c were confirmed in two independent alleles by sequencing. These two alleles were maintained over TM3, Ser. However, very few homozygous mutant flies emerged from breeding of either strain (4 homozygotes out of 457 flies and 5 out of 455 , respectively), suggesting that deletion of these two exons severely impaired adult viability. This finding is consistent with previous studies showing that Syp $^{\mathrm{f} 03775}$ and Syp $^{\mathrm{e} 00286}$ flies have decreased adult viability 28. Notably, insertion sites in $S y p^{\mathrm{f03} 775}$ and $S y p^{\mathrm{e} 00286}$ are within the C-terminal region of Syp and specifically, f03775 is located in the intronic region between the two exons deleted in our deletion lines and e00286 is located upstream of these two exons.

\section{Discussion}

Starvation-Induced Hyperactivity (SIH) has been observed in different species 3.12 , suggesting that it has a genetic component. SIH facilitates food acquisition and energy intake when food is available [7, but it increases energy expenditure and makes starved 
animals even more vulnerable when food is not available 44. Therefore, genetic dispositions to either too much or too little SIH would impair fitness depending on the environment. Taking advantage of the genetic diversity in the DGRP strains derived from a wild population, we have demonstrated for the first time the significant genetic contribution to SIH. Our studies show that the broad sense heritability $\left(H^{2}\right)$ of SIH is 0.38 , which is lower than the reported heritability of developmental traits in the DGRP (e.g. $H^{2}$ is 0.89 for developmental time [36], 0.66-0.88 for pigmentation [37, and 0.71-0.78 wing morphology [38]), but is higher than the reported heritability of behavioral traits in the DGRP (e.g. $H^{2}$ is 0.03-0.09 for courtship behavior 39 and 0.02-0.45 for olfactory behavior $[40-42]$ ), suggesting that SIH has a relatively strong genetic basis compared to other behavioral traits.

Our genome-wide association studies identified 23 loci from 14 genes significantly associated with SIH in adult Drosophila. Gene enrichment analyses revealed that mRNA binding proteins were important for SIH. We validated the role of RNA-binding protein Syp in adult neurons in regulating SIH and showed that it is likely that specific Syp splice variants were responsible for SIH. Although the present study is the first unbiased genome-wide study on SIH, other candidate gene approaches have discovered a number of SIH genes in Drosophila in recent years, which includes AMP-activated protein kinase (AMPK) [5, 6], Adipokinetic hormone (Akh) 4, Adipokinetic hormone receptor $(A k h R)$ [8], Insulin-like peptides (Ilps) [8, Insulin-like receptor (InR) 8], Tyramine beta hydroxylase (Tbh) [7], and $d G 9 a[9]$. Majority of these candidates play important roles in relaying metabolic information to neurons. Expression of some of these genes were regulated by starvation, as shown in our differential expression analyses from RNA-seq data. For example, the mRNA levels of both $A k h R$ and InR were significantly altered after starvation $(\log \mathrm{FC}=-1.08$ and $\log \mathrm{FC}=1.45 ; P=$ $1.65 \mathrm{e}-9$, and $P=3.15 \mathrm{e}-23$ for $A k h R$ and $\operatorname{InR}$, respectively. S6 Table. The present study further suggests that RBPs and post-transcriptional regulation could be another important pathway that regulates SIH. Moreover, our RNA-seq data show that only 15 genes ( 3 up-regulated and 12 down-regulated) are shared between the 658 differentially expressed genes and the 180 alternatively spliced genes, suggesting that regulation at both transcriptional and post-transcriptional levels for the same gene is uncommon in response to starvation, at least in the head. Gene enrichment analyses further suggest that metabolic genes tend to be regulated at the transcriptional level while genes involved in the mRNA regulation tend to be regulated post-transcriptionally (S6 Fig). This is also shown in our splice variants analysis followed by GO analysis. Genes encoding RBPs seem to be especially regulated by alternative splicing (S8 Fig).

Syp has a number of splice variants (Fig $5 \mathrm{~B}$ ). These variants mainly differ at the $\mathrm{N}$-terminus and the $\mathrm{C}$-terminus, whereas the remaining the middle region of the protein, corresponding to the three RNA recognition motifs, are largely unchanged. The sequence discrepancy among various splice variants implies a potential role of UTRs in response to different stimuli. Our results from RNA-seq demonstrate that under starvation, Syp is mainly regulated at the C-terminus (Fig 5A). We postulate that either Syp itself or some other RBPs may regulate the splicing of Syp under starvation. Such self-regulatory or cross-regulatory networks in the alternative splicing of RBPs have been reported in previous studies 43 . Moreover, alternatively spliced exons in Syp seem not only involved in regulating starvation responses. We have found that deletion of these axons leads to severely impaired adult viability. It is likely that these alternatively spliced exons play different roles at different stages.

Syp is a Drosophila homolog of human SYNaptotagmin-binding Cytoplasmic RNA-Interacting Protein (SYNCRIP)/hnRNP Q, and they share $47 \%$ sequence identity [28]. Similar to the mammalian SYNCRIP, Syp consists of three RNA recognition motifs and one acidic domain at the N-terminus 28,44. However, it lacks 
the arginine-glycine-glycine domain at the C-terminus [28. In mammals, different domains mediate the interaction of SYNCRIP with different effectors, which renders SYNCRIP a wide range of functions including circadian regulation [45 47, neuronal morphogenesis [48 50], and stress response [51]. Misregulation of SYNCRIP has been reported in neurodegenerative diseases [52,53], psychiatric disorders [54,55], and cancer 56, 57]. In flies, studies have shown that Syp is required for different developmental phenotypes, such as oogenesis [28], maintaining a normal structure and function of synapses at the neuromuscular junction in larvae [29, 30], and determining neuron/glia cell fates 31, 32. The present finding is the first indication that Syp has a unique function in adult flies (Fig 3) independent of its developmental effects (Fig 4 ).

At the subcellular and molecular level, previous studies have shown that Syp, in muscle cells at the Drosophila larval neuromuscular junction, can modulate the presynaptic vesicle release through regulating postsynaptic translation of a retrograde signal 30 ] and can regulate activity-dependent synaptic plasticity 58. Similar functions have also been observed in mammalian SYNCRIP such that it is a component of neuronal RNA transport granules that can regulate dendritic morphology [48,59 61], and that it inhibits translation by competing with the poly(A) binding protein 62 . We hypothesize that Syp might be able to quickly affect protein synthesis in neurons and even local protein synthesis in synapses in response to starvation. Further studies are needed to examine the molecular mechanisms, including the upstream and downstream pathways associated with Syp in adult neurons in response to starvation.

In summary, we report genome-wide association studies of SIH as a behavioral trait. Our data suggest a strong genetic component in SIH. We further report an essential role of Syp and post-transcriptional regulation in adult neurons in regulating SIH.

\section{Materials and methods}

\section{Drosophila stocks}

The Drosophila melanogaster Genetic Reference Panel (DGRP) strains were from the Bloomington Drosophila Stock Center (BDSC). The UAS- Syp RNAi lines (\#33011, \#33012) and the genetic control line were from the Vienna Drosophila Resource Center (VDRC). The actin-Gal4 (\#4414) and the pan-neuronal driver nSyb-Gal4 (\#51635) were from the BDSC. Unless stated otherwise, all flies were reared on the standard cornmeal medium from FlyKitchen at the University of Chicago at $25{ }^{\circ} \mathrm{C}$ under a 12-hr:12-hr light: dark cycle with the light on at 06:00 and off at 18:00.

\section{Locomotor assay setup}

Male flies, 1 to $3 \mathrm{~d}$ old, were anesthetized briefly and transferred into activity tubes filled with the standard cornmeal medium where they were allowed to recover from CO2. One day later, flies were randomly separated into two groups. Flies in the first group were transferred into activity tubes filled with $4 \%$ sucrose plus $5 \%$ yeast in $1 \%$ agar (food condition), and flies in the second group were transferred into activity tubes filled with $1 \%$ agar only (starvation condition). The other end of activity tubes was inserted with a small cotton ball to allow air exchange for the fly and also to prevent fly from escaping. The locomotor activity of each fly was monitored using the Drosophila Activity Monitor (DAM2, Trikinetics Inc.). Unless stated otherwise, the assay chamber was maintained at $25{ }^{\circ} \mathrm{C}$ under a 12-hr:12-hr light: dark cycle with the light on at 06:00 and off at 18:00. The start point on the plots is the light off point, which is 18:00 on the setup day, which is about 6-7 hours after flies were transferred to the activity tubes. We used 8 flies per condition per genotype for screening. The number of flies in other 
experiments was indicated in the text.

347

\section{Data analysis of the locomotor responses to starvation}

Infrared beam breaks per 30-minute interval from two conditions were averaged and plotted as a function of time point for each strain. The total activity of each fly from a 12-hour period (either daytime or nighttime) in each condition was first summed, and the activity difference between the two conditions was then calculated. Since flies were monitored for a total of sixty hours, there were five DAs. The largest DA among five DAs was referred to as DA of each fly. The averaged DA from 8 flies was used to represent the strain DA.

\section{Starvation Resistance}

Starvation Resistance is represented by the time point (0-120) when all eight flies under starvation were dead. The death of a fly was inferred from the activity data such that there were no beam crosses. In the case of all flies survived to the last time point, an index of 121 was used.

\section{Genome-wide association analysis}

Genome-wide association analysis on SIH was performed using the DGRP analysis pipeline at http://dgrp2.gnets.ncsu.edu 16 17]. Briefly, each raw dataset was first adjusted for the Wolbachia infection and the inversion with a linear model where the raw dataset as the response variable and the infection status and five major inversion polymorphisms as covariates (S3 Table). Residuals from this linear model were then used as response variable to fit a mixed linear model: $\mathrm{Y}=\mu+\mathrm{G}+\mathrm{r}$, where $\mu$ is the overall population mean for each trait, $G$ is the effect of SNPs or INDELs being tested, and $\mathrm{r}$ is a polygenic component with covariance between lines determined by their genome relationship 17]. A total of 1,905,103 SNPs or INDELS with minor allele frequencies (MAFs) larger than 0.05 were included in the analysis. A nominal $P$-value threshold of $P<1 \times 10^{-5}$ was used for declaring SNPs or INDELs to be significantly associated with trait variation by following previous studies 16,17 .

\section{Gene enrichment analysis}

The web-based enrichment analysis tool FlyEnrichr 24,25] was applied to evaluate the list of candidate genes, and to obtain a set of enriched functional annotations in three domains: biological process (BP), cellular component (CC), and molecular function (MF) 63 or in KEGG (Kyoto Encyclopedia of Genes and Genomes) pathways 64. GO (gene ontology) or KEGG terms with $P<0.05$ (after Benjamini-Hochberg correlation) were listed in S5 Table and S8 Table.

\section{Western blotting}

For the Gal80 ${ }^{\text {ts }}$ experiment, a group of 25-30 male flies, 1 to $3 \mathrm{~d}$ old, were reared on the standard cornmeal medium at either $18^{\circ} \mathrm{C}$ or $31^{\circ} \mathrm{C}$ for two days. For the protein level of Syp in $w^{1118}$ flies under either food or starvation conditions, a group of 25-30 male flies, 1 to $3 \mathrm{~d}$ old, were maintained in either food or starvation conditions at the room temperature for one day. The total protein from adult heads was then extracted and quantified. A total of $50 \mu \mathrm{g}$ protein from each sample was loaded for SDS-PAGE.

Separated proteins were electrophoretically transferred to the PVDF membrane. After blocking, the membrane was incubated with primary antibodies (Guinea pig anti-Syp 
1:2000 Ilan Davis, UK; Mouse anti-tubulin 1:500 Developmental Studies Hybridoma Bank, DSHB) and then the secondary antibodies (peroxidase-labeled anti-Guinea pig IgG 1:10,000, Sigma; peroxidase-labeled anti-mouse IgG 1:10,000, Jackson ImmunoResearch Laboratories). Signals were detected with enhanced chemiluminescence (ThermoScientific).

\section{RNA extraction}

A group of 30 male flies, 1 to $3 \mathrm{~d}$ old, were maintained under either food or starvation conditions for one day. Four biological samples were collected for each condition. Total RNA was extracted from heads using RNA extraction kit (Zymo Research). After the removal of genomic DNA (DNA-free kit, AMBION), total RNA was quantified using a NanoDrop spectrophotometer (Thermo Scientific), and the concentrations of total RNA were then diluted to $100 \mathrm{ng} / \mu \mathrm{L}$.

\section{RNA sequencing and data analysis}

RNA sequencing was performed in the Genomics Core Facility at the University of Chicago. Briefly, the integrity of total RNA was assessed using a bioanalyzer 2100 (Agilent), and poly(A)+ RNA-seq libraries were then prepared from each sample, multiplexed, and sequenced by 100-bp paired ends using Illumina HiSeq4000 sequencer S3 Fig S4 Fig). Sequencing was duplicated using two flow cells. A total of 15-30 million reads were generated for each library per sequencing batch. Sequencing quality was assessed using FastQC [65], and the mean quality score ranges from 38.84 to 39 . Raw sequencing reads from each group (treatments + biological replicates + sequencing batches) were individually mapped to the Drosophila genome (v6.22) using STAR (v2.6.1b) 66. About $95 \%$ of raw reads from each sample were uniquely mapped to the genome. Each bam file was then processed using ASpli 67 to obtain differentially expressed genes and thereby to access the sequencing batch effect (S5 Fig). Since results from two sequencing batches are highly correlated $(\rho=0.99, P<2.2 \mathrm{e}-16$, Pearson correlation test), we merged two sequencing bam files from the same sample using samtools 68] for downstream analyses. Alternative splice variants were analyzed using Leafcutter [33].

\section{RT-qPCR}

The cDNAs were synthesized using SMARTScribe Reverse Transcription Kit (TaKaRa). Two pairs of primers that target either both exons b and c or exon b only were used to amplify these two exons. Primer sequences of the first pair of primers are: 5'- TTC ACC GAT GGC TAG TGG AC and 5'- GTT GGC CAA CGA CTC TGC CA, and primer sequences of the second pair of primers are: 5'- TTC GGT TTC TCG GAC TAT CG and 5' - CCA CCG TTC GGG TAA TCA TA. One pair of primers that targeted the house-keeping gene ribosomal protein 49 (rp 49) was used as the internal control for qPCR. Primer sequences are: 5'- GCT AAG CTG TCG CAC AAA TG and 5'- GTT CGA TCC GTA ACC GAT GT.

\section{Generation of exon b and exon c deletion using CRISPR/Cas9}

Two different deletion alleles were generated using CRISPR/Cas9 $[34,35]$. The sgRNAs were designed using FlyCRISPR Optimal Target Finder

(http://targetfinder.flycrispr.neuro.brown.edu/) and in vitro transcribed using T7

transcript kit (MEGAscript T7 Transcription Kit, Cat \# AMB13345). The sequences of sgRNAs were shown in S9 Fig. The sgRNAs and Cas9 protein (RNA bio company, Cat 
\#CP01) were injected into embryos from flies with a genotype: nos-phiC31, y[1] sc[1] v[1] sev[21]; Py[+t7. 7]=CaryPattP2 (BDSC \#25710). Deletion alleles were maintained over TM3, Ser (BDSC \#4534). Deletion was confirmed by sequencing the targeted region amplified using a pair of primers (S9 Fig). Primer sequences are 5'- CGA ACT CTC TGT GTC GCA AG (genoF) and 5'- AGT TGG CAT TGG ATT GGT GT (genoR).

\section{Correlation, quantitative genetic, and statistical analyses}

All analyses were performed using the statistical software $\mathrm{R}$ v3.6.1

(http://www.r-project.org). Pearson correlation test was applied for correlation analyses. Other statistical tests were indicated in the text or figure legends. The broad-sense heritability $\left(H^{2}\right)$ of $\mathrm{SIH}$ was computed as $H^{2}=\sigma_{\mathrm{G}}^{2} /\left(\sigma^{2}{ }_{\mathrm{G}}+\sigma^{2}{ }_{\mathrm{E}}\right)$, where $\sigma^{2}{ }_{\mathrm{G}}$ is the among-line variance component and $\sigma^{2}{ }_{\mathrm{E}}$ is the error variance.

\section{Data availability}

All raw and processed RNA-sequencing data generated in this study have been submitted to the NCBI Gene Expression Omnibus (GEO) under accession number GSE149790.

\section{Supporting information}

S1 Fig. Setup image of the locomotor activity assay. One end of the activity tube (Diameter $\times$ Length $=5 \mathrm{~mm} \times 65 \mathrm{~mm}$, Trikinetics Inc.) was filled with either $4 \%$ sucrose plus $5 \%$ yeast in $1 \%$ agar in the food condition or $1 \%$ agar only in the starvation condition. The length of the medium is $1 \mathrm{~cm}$. The other end of the tube was stopped with a small cotton ball. One male fly was introduced into each tube.

S2 Fig. Linkage Disequilibrium map and Quantile-Quantile plot for GWA on SIH.

S3 Fig. Experimental design and workflow of RNA sequencing.

S4 Fig. Integrity of total RNA from each sample.

S5 Fig. Correlation analyses of genes from two sequencing batches. A: Scatter plot for all genes. A total of 9452 genes were included in the analysis. Each dot represents one gene. Dots with cyan color are the genes that show the same direction of change after starvation for two batches. Dots with yellow color are the genes that show the opposite direction of change after starvation for two batches. B: Scatter plot for genes with false discovery rate $(\mathrm{FDR})<0.05$ and fold change $\geq 2$ or fold change $\leq-2$.

S6 Fig. Differential expression analysis. A: Clustering and heatmap showing mRNA levels in four biological replicates of each condition. B: Volcano plot of significantly regulated genes by starvation (Cutoff values: FDR $<0.05$ and fold change $\geq 2$ or fold change $\leq-2$ ). (C) Enriched KEGG pathways associated with either down-regulated (blue bars) or up-regulated (red bars) genes. 
S7 Fig. Western blot and quantification of Syp protein level. A: Western blot of adult fly head homogenate from $w^{1118}$ flies under either food or starvation conditions. Four biological replicates per condition. Tubulin is the loading control. B: Quantification of Syp from panel A. n.s: $P>0.05$, unpaired $t$-test. Error bars represent SEM.

S8 Fig. Molecular Function GO terms associated with alternatively spliced genes. Only terms associated with more than 4 genes were shown in this plot. See the complete list in S8 Table.

S9 Fig. Generation of Syp exons b and c deletion lines using CRISPR/Cas9. Two sgRNAs that target the intronic regions adjacent to exon $b$ and exon c (symbol $\mathrm{X}$ in red) cut each side of the targeted region, respectively. The sequences of sgRNAs were show in the rectangles. A pair of primers (genoF and genoR) were designed to amplify the targeted region for sequencing. Based on the sequencing results, two different deletion alleles were generated. Exons b and c were deleted in both alleles.

S1 Table. Data of SIH and of Starvation Resistance for DGRP strains.

S2 Table. Analysis of variance of SIH in the DGRP.

S3 Table. Covariates analyses.

S4 Table. Gene lists of GWA with $P<1 \times 10^{-4}, P<8 \times 10^{-5}, P<6 \times$ $10^{-5}, P<4 \times 10^{-5}$, and $P<2 \times 10^{-5}$.

S5 Table. Over-representation of Gene Ontology Categories associated with nominated genes with $P<1 \times 10^{-4}$ or $P<8 \times 10^{-5}$.

S6 Table. Differentially expressed genes.

S7 Table. Alternatively spliced genes.

S8 Table. Over-representation of Gene Ontology Categories and KEGG pathways associated with alternatively spliced genes.

\section{Acknowledgments}

We thank Ilan Davis for sharing the anti-Syp antibody. We thank Chunyu Liu and Xiaochang Zhang for helpful discussions. We thank Yang I Li, Jack Humphrey, Julian Beckman, Shuaibo Han, Wei A Du, and Meredith Wells for technical assistance. We thank the Bloomington Drosophila Stock Center (BDSC) and the Vienna Drosophila Resource Center (VDRC) for fly stocks. The computational analysis in this study was performed with resources provided by the University of Chicago Research Computing Center. This work was supported by the National Institutes of Health (T32MH020065 \& T32DA434693 to W.C., R01GM100768 to X.Z.).

3
4
5
6
7
8
9
30
31
32
33
34
35
36

487 


\section{References}

1. McCue MD. Starvation physiology: reviewing the different strategies animals use to survive a common challenge. Comparative Biochemistry and Physiology Part A: Molecular \& Integrative Physiology. 2010;156(1):1-18.

2. Zhang D, Xiao Z, Zeng B, Li K, Tang Y. Insect behavior and physiological adaptation mechanisms under starvation stress. Frontiers in physiology. 2019;10:163.

3. Connolly K. Locomotor activity in Drosophila as a function of food deprivation. Nature. 1966;209(5019):224.

4. Lee G, Park JH. Hemolymph sugar homeostasis and starvation-induced hyperactivity affected by genetic manipulations of the adipokinetic hormone-encoding gene in Drosophila melanogaster. Genetics. 2004;167(1):311-323.

5. Johnson EC, Kazgan N, Bretz CA, Forsberg LJ, Hector CE, Worthen RJ, et al. Altered metabolism and persistent starvation behaviors caused by reduced AMPK function in Drosophila. PLoS One. 2010;5(9):e12799.

6. Braco JT, Gillespie EL, Alberto GE, Brenman JE, Johnson EC. Energy-dependent modulation of glucagon-like signaling in Drosophila via the AMP-activated protein kinase. Genetics. 2012;192(2):457-466.

7. Yang Z, Yu Y, Zhang V, Tian Y, Qi W, Wang L. Octopamine mediates starvation-induced hyperactivity in adult Drosophila. Proceedings of the National Academy of Sciences. 2015;112(16):5219-5224.

8. Yu Y, Huang R, Ye J, Zhang V, Wu C, Cheng G, et al. Regulation of starvation-induced hyperactivity by insulin and glucagon signaling in adult Drosophila. Elife. 2016;5:e15693.

9. Shimaji K, Tanaka R, Maeda T, Ozaki M, Yoshida H, Ohkawa Y, et al. Histone methyltransferase G9a is a key regulator of the starvation-induced behaviors in Drosophila melanogaster. Scientific reports. 2017;7(1):14763.

10. Pirke KM, Broocks A, Wilckens T, Marquard R, Schweiger U. Starvation-induced hyperactivity in the rat: the role of endocrine and neurotransmitter changes. Neuroscience \& Biobehavioral Reviews. 1993;17(3):287-294.

11. Chen D, Steele AD, Lindquist S, Guarente L. Increase in activity during calorie restriction requires Sirt1. Science. 2005;310(5754):1641-1641.

12. Zhao ZJ, Chen KX, Liu YA, Wang CM, Cao J. Decreased circulating leptin and increased neuropeptide $\mathrm{Y}$ gene expression are implicated in food deprivation-induced hyperactivity in striped hamsters, Cricetulus barabensis. Hormones and behavior. 2014;65(4):355-362.

13. Dutertre M, Sanchez G, Barbier J, Corcos L, Auboeuf D. The emerging role of pre-messenger RNA splicing in stress responses: sending alternative messages and silent messengers. RNA biology. 2011;8(5):740-747.

14. Pleiss JA, Whitworth GB, Bergkessel M, Guthrie C. Rapid, transcript-specific changes in splicing in response to environmental stress. Molecular cell. 2007;27(6):928-937. 
15. Salati LM, Amir-Ahmady B. Dietary regulation of expression of glucose-6-phosphate dehydrogenase. Annual review of nutrition. 2001;21(1):121-140.

16. Mackay TF, Richards S, Stone EA, Barbadilla A, Ayroles JF, Zhu D, et al. The Drosophila melanogaster genetic reference panel. Nature. 2012;482(7384):173.

17. Huang W, Massouras A, Inoue Y, Peiffer J, Ràmia M, Tarone AM, et al. Natural variation in genome architecture among 205 Drosophila melanogaster Genetic Reference Panel lines. Genome research. 2014;24(7):1193-1208.

18. Mackay TF, Huang W. Charting the genotype-phenotype map: lessons from the Drosophila melanogaster Genetic Reference Panel. Wiley Interdisciplinary Reviews: Developmental Biology. 2018;7(1):e289.

19. Marygold SJ, Leyland PC, Seal RL, Goodman JL, Thurmond J, Strelets VB, et al. FlyBase: improvements to the bibliography. Nucleic acids research. 2012;41(D1):D751-D757.

20. McGuire SE, Mao Z, Davis RL. Spatiotemporal gene expression targeting with the TARGET and gene-switch systems in Drosophila. Sci STKE. 2004;2004(220):pl6-pl6.

21. Hall J. Genetics and molecular biology of rhythms in Drosophila and other insects. Advances in genetics. 2003;48:1.

22. Lee YCG, Yang Q, Chi W, Turkson SA, Du WA, Kemkemer C, et al. Genetic architecture of natural variation underlying adult foraging behavior that is essential for survival of Drosophila melanogaster. Genome biology and evolution. 2017;9(5):1357-1369.

23. Harbison ST, McCoy LJ, Mackay TF. Genome-wide association study of sleep in Drosophila melanogaster. BMC genomics. 2013;14(1):281.

24. Chen EY, Tan CM, Kou Y, Duan Q, Wang Z, Meirelles GV, et al. Enrichr: interactive and collaborative HTML5 gene list enrichment analysis tool. BMC bioinformatics. 2013;14(1):128.

25. Kuleshov MV, Jones MR, Rouillard AD, Fernandez NF, Duan Q, Wang Z, et al. Enrichr: a comprehensive gene set enrichment analysis web server 2016 update. Nucleic acids research. 2016;44(W1):W90-W97.

26. Chen D, Qu C, Bjorum SM, Beckingham KM, Hewes RS. Neuronal remodeling during metamorphosis is regulated by the alan shepard (shep) gene in Drosophila melanogaster. Genetics. 2014;197(4):1267-1283.

27. Brand AH, Perrimon N. Targeted gene expression as a means of altering cell fates and generating dominant phenotypes. development. 1993;118(2):401-415.

28. McDermott SM, Meignin C, Rappsilber J, Davis I. Drosophila Syncrip binds the gurken mRNA localisation signal and regulates localised transcripts during axis specification. Biology open. 2012;1(5):488-497.

29. McDermott SM, Yang L, Halstead JM, Hamilton RS, Meignin C, Davis I. Drosophila Syncrip modulates the expression of mRNAs encoding key synaptic proteins required for morphology at the neuromuscular junction. Rna. 2014;20(10):1593-1606. 
30. Halstead JM, Lin YQ, Durraine L, Hamilton RS, Ball G, Neely GG, et al. Syncrip/hnRNP Q influences synaptic transmission and regulates BMP signaling at the Drosophila neuromuscular synapse. Biology open. 2014;3(9):839-849.

31. Liu Z, Yang CP, Sugino K, Fu CC, Liu LY, Yao X, et al. Opposing intrinsic temporal gradients guide neural stem cell production of varied neuronal fates. Science. 2015;350(6258):317-320.

32. Syed MH, Mark B, Doe CQ. Steroid hormone induction of temporal gene expression in Drosophila brain neuroblasts generates neuronal and glial diversity. Elife. 2017;6:e26287.

33. Li YI, Knowles DA, Humphrey J, Barbeira AN, Dickinson SP, Im HK, et al. Annotation-free quantification of RNA splicing using LeafCutter. Nature genetics. 2018;50(1):151.

34. VanKuren NW, Long M. Gene duplicates resolving sexual conflict rapidly evolved essential gametogenesis functions. Nature ecology \& evolution. 2018;2(4):705-712.

35. Bassett AR, Liu JL. CRISPR/Cas9 and genome editing in Drosophila. Journal of genetics and genomics. 2014;41(1):7-19.

36. Horváth B, Betancourt AJ, Kalinka AT. A novel method for quantifying the rate of embryogenesis uncovers considerable genetic variation for the duration of embryonic development in Drosophila melanogaster. BMC evolutionary biology. 2016;16(1):200.

37. Dembeck LM, Huang W, Magwire MM, Lawrence F, Lyman RF, Mackay TF. Genetic architecture of abdominal pigmentation in Drosophila melanogaster. PLoS genetics. 2015;11(5):e1005163.

38. Vonesch SC, Lamparter D, Mackay TF, Bergmann S, Hafen E. Genome-wide analysis reveals novel regulators of growth in Drosophila melanogaster. PLoS genetics. 2016;12(1):e1005616.

39. Gaertner BE, Ruedi EA, McCoy LJ, Moore JM, Wolfner MF, Mackay TF. Heritable variation in courtship patterns in Drosophila melanogaster. G3: Genes, Genomes, Genetics. 2015;5(4):531-539.

40. Swarup S, Huang W, Mackay TF, Anholt RR. Analysis of natural variation reveals neurogenetic networks for Drosophila olfactory behavior. Proceedings of the National Academy of Sciences. 2013;110(3):1017-1022.

41. Brown E, Layne J, Zhu C, Jegga A, Rollmann S. Genome-wide association mapping of natural variation in odour-guided behaviour in Drosophila. Genes, Brain and Behavior. 2013;12(5):503-515.

42. Arya GH, Magwire MM, Huang W, Serrano-Negron YL, Mackay TF, Anholt RR. The genetic basis for variation in olfactory behavior in Drosophila melanogaster. Chemical senses. 2015;40(4):233-243.

43. Brooks AN, Duff MO, May G, Yang L, Bolisetty M, Landolin J, et al. Regulation of alternative splicing in Drosophila by 56 RNA binding proteins. Genome research. 2015;25(11):1771-1780.

44. Beuck C, Williamson JR, Wüthrich K, Serrano P. The acidic domain is a unique structural feature of the splicing factor SYNCRIP. Protein Science.

2016;25(8):1545-1550. 
45. Kim DY, Kwak E, Kim SH, Lee KH, Woo KC, Kim KT. hnRNP Q mediates a phase-dependent translation-coupled mRNA decay of mouse Period3. Nucleic acids research. 2011;39(20):8901-8914.

46. Lee KH, Woo KC, Kim DY, Kim TD, Shin J, Park SM, et al. Rhythmic interaction between Period1 mRNA and hnRNP Q leads to circadian time-dependent translation. Molecular and cellular biology. 2012;32(3):717-728.

47. Lim I, Jung Y, Kim DY, Kim KT. HnRNP Q has a suppressive role in the translation of mouse cryptochrome1. PloS one. 2016;11(7):e0159018.

48. Chen HH, Yu HI, Chiang WC, Lin YD, Shia BC, Tarn WY. hnRNP Q regulates Cdc42-mediated neuronal morphogenesis. Molecular and cellular biology. 2012;32(12):2224-2238.

49. Xing L, Yao X, Williams KR, Bassell GJ. Negative regulation of RhoA translation and signaling by hnRNP-Q1 affects cellular morphogenesis. Molecular biology of the cell. 2012;23(8):1500-1509.

50. Williams KR, McAninch DS, Stefanovic S, Xing L, Allen M, Li W, et al. hnRNP-Q1 represses nascent axon growth in cortical neurons by inhibiting Gap-43 mRNA translation. Molecular biology of the cell. 2016;27(3):518-534.

51. Kim D, Kim W, Lee K, Kim SH, Lee H, Kim H, et al. hnRNP Q regulates translation of p53 in normal and stress conditions. Cell Death \& Differentiation. 2013;20(2):226-234.

52. Mourelatos Z, Abel L, Yong J, Kataoka N, Dreyfuss G. SMN interacts with a novel family of hnRNP and spliceosomal proteins. The EMBO journal. 2001;20(19):5443-5452.

53. Rizzo F, Nizzardo M, Vashisht S, Molteni E, Melzi V, Taiana M, et al. Key role of SMN/SYNCRIP and RNA-Motif 7 in spinal muscular atrophy: RNA-Seq and motif analysis of human motor neurons. Brain. 2019;142(2):276-294.

54. Lelieveld SH, Reijnders MR, Pfundt R, Yntema HG, Kamsteeg EJ, de Vries P, et al. Meta-analysis of 2,104 trios provides support for 10 new genes for intellectual disability. Nature neuroscience. 2016;19(9):1194.

55. Guo H, Duyzend MH, Coe BP, Baker C, Hoekzema K, Gerdts J, et al. Genome sequencing identifies multiple deleterious variants in autism patients with more severe phenotypes. Genetics in Medicine. 2019;21(7):1611-1620.

56. Vu LP, Prieto C, Amin EM, Chhangawala S, Krivtsov A, Calvo-Vidal MN, et al. Functional screen of MSI2 interactors identifies an essential role for SYNCRIP in myeloid leukemia stem cells. Nature genetics. 2017;49(6):866.

57. Gachet S, El-Chaar T, Avran D, Genescà E, Catez F, Quentin S, et al. Deletion 6q drives T-cell leukemia progression by ribosome modulation. Cancer discovery. 2018;8(12):1614-1631.

58. Titlow J, Robertson F, Järvelin A, Ish-Horowicz D, Smith C, Gratton E, et al. Syncrip/hnRNP Q is required for activity-induced Msp300/Nesprin-1 expression and new synapse formation. Journal of Cell Biology. 2020;219(3). 
59. Bannai H, Fukatsu K, Mizutani A, Natsume T, Iemura Si, Ikegami T, et al. An RNA-interacting protein, SYNCRIP (heterogeneous nuclear ribonuclear protein Q1/NSAP1) is a component of mRNA granule transported with inositol 1, 4, 5 -trisphosphate receptor type $1 \mathrm{mRNA}$ in neuronal dendrites. Journal of Biological Chemistry. 2004;279(51):53427-53434.

60. Kanai Y. Dohmae N, Hirokawa N. Kinesin transports RNA: isolation and characterization of an RNA-transporting granule Neuron. 2004;43:513-525.

61. Elvira G, Wasiak S, Blandford V, Tong XK, Serrano A, Fan X, et al. Characterization of an RNA granule from developing brain. Molecular \& cellular proteomics. 2006;5(4):635-651.

62. Svitkin YV, Yanagiya A, Karetnikov AE, Alain T, Fabian MR, Khoutorsky A, et al. Control of translation and miRNA-dependent repression by a novel poly (A) binding protein, hnRNP-Q. PLoS biology. 2013;11(5):e1001564.

63. Dolinski K, Dwight S, Eppig J, Harris M, Hill D, Issel-Tarver L, et al. Gene ontology: tool for the unification of biology. The Gene Ontology Consortium. Nat Genet. 2000;25(1):2529Attri.

64. Ogata H, Goto S, Sato K, Fujibuchi W, Bono H, Kanehisa M. KEGG: Kyoto encyclopedia of genes and genomes. Nucleic acids research. 1999;27(1):29-34.

65. Andrews S, et al.. FastQC: a quality control tool for high throughput sequence data; 2010.

66. Dobin A, Davis C, Schlesinger F, Drenkow J, Zaleski C, Jha S, et al.. STAR: ultrafast universal RNA-seq aligner. Bioinformatics29, 15-21; 2013.

67. Mancini E, Iserte J, Yanovsky M, Chernomoretz A. ASpli: analysis of alternative splicing using RNA-Seq. Bioconductor: R package version. 2017;1(3).

68. Li H, Handsaker B, Wysoker A, Fennell T, Ruan J, Homer N, et al. .(2009): The Sequence Alignment/Map format and SAMtools. Bioinformatics.

2009;25(16):2078-2079. 